\title{
Design of Large Torque Micro-Nano Platform Based on Piezoelectric Stack
}

\author{
Ke Liang, Hong Jin a, ${ }^{*}$ and Yueping Mo \\ School of Hydraulic, Energy and Power Engineering Yangzhou University Yangzhou 225127 \\ China \\ alianglvd@163.com
}

Keywords: Micro-nano Platform, Piezoelectric stack, Triangle amplification.

\begin{abstract}
With the development of precision engineering and precision instruments, the micro-nano platform becomes the important carrier in the precision positioning technology, which provides large torque and high precision. In this paper, a kind of large torque micro-nano platform is designed and created, which apply piezoelectric stack and triangle amplification principle. Theoretical analysis calculates the displacement amplification of drive mechanism and the displacement and stress variation of platform. The characteristics analysis shows that the micro-nano platform can provide large output displacement stably and quickly, and it has very strong practical.
\end{abstract}

\section{Introduction}

Into the 90's, whether it is optoelectronic devices or integrated circuit processing or cell manipulation in biomedical engineering, more and more requirements are put forward for large stroke, high precision, small volume and fast response. At the same time the requirements for positioning accuracy and resolution are increased to the nanometer scale $[1,2]$.

Piezoelectric ceramic actuator is widely used in micro-nano positioning system due to its advantage, such as high displacement resolution, simple structure, low heat, small volume, high rigidity, fast response, free from magnetic interference, no wear, no lubrication, etc. However, piezoelectric actuator also has a small displacement, hysteresis, non-linear, creep and other shortcomings [3]. In order to enlarge the displacement, which is currently enlarged by using hinge form [4 8], but the output torque decreases. In order to solve this problem, an amplification mechanism based on piezoelectric stack and triangular amplification principle is designed to realize the driving of micro-nano positioning system. And on the basis of this theory, a kind of micro-nano platform is designed, which not only owns higher displacement accuracy and output torque, but also owns larger output displacement.

\section{Structure and Principle of Large Torque Micro-Nano Platform based on Piezoelectric Stack}

The micro-nano platform composed of piezoelectric stack and triangular amplification principle is shown in Fig.1. The Fig.1 (a) is physical picture, and the Fig.1 (b) is the model diagram. The working principle of this micro-nano platform is that the amplification mechanism deforms after applying the forward voltage on the piezoelectric stack, the deformation produced by the magnifying mechanism is realized by connecting the hinge to drive the platform to realize the corresponding displacement, when one end of the magnifying mechanism is fixed to the platen by a fixed hinge. The auxiliary hinge only assists the platform in moving smoothly and evenly. 


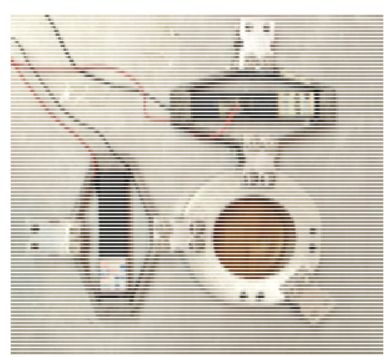

(a) Physical picture

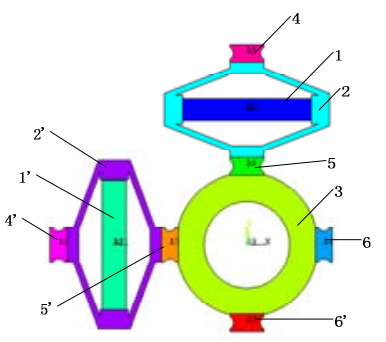

(b) Model diagram

Fig. 1 The structure diagram of micro-nano platform

1, 1'-Piezoelectric stack; 2, 2'-stack Amplifying mechanism; 3-platform; 4, 4'-Fixed hinge;

5, 5'-Connected hinges; 6,6 '-Auxiliary hinge

\section{Principle of Piezoelectric Stack Displacement Amplifying Actuator based on Triangle Magnification Principle}

Piezoelectric stack displacement amplifying actuator based on the triangulation principle is composed of an amplification mechanism and a piezoelectric stack.

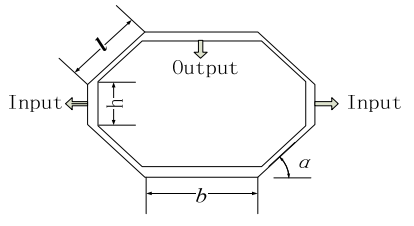

(a)Schematic

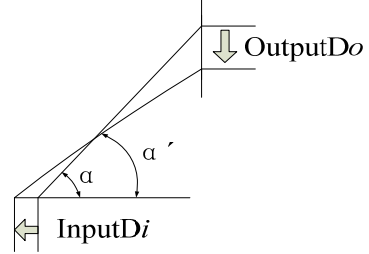

(b) Quarter model diagram

Fig.2 The structure diagram of amplification mechanism

Amplifying mechanism adopts triangular amplification principle, and its structure is shown in Fig.2 (a). The structure is that eight links are connected end to end, when an input displacement of the illustrated direction is applied; the corresponding output is shifted in the vertical direction. Fig.2 (b) is a quarter of the model of the amplification mechanism. Input displacement is Di and output displacement is Do, and the relationship between them is as follow:

$$
\left\{\begin{array}{l}
l \cos \alpha+D i=l \cos \alpha^{\prime} \\
l \sin \alpha-D o=l \sin \alpha^{\prime}
\end{array}\right.
$$

In which, 1 is the length of link; $\alpha$ is the angle between link and the horizontal position; $\alpha^{\prime}$ is the deflection angle after input displacement.

Solving Equations (1), the two sides of the two equations are squared simultaneously, and then corresponding item is summed, then $\alpha^{\prime}$ is eliminated, the equation is:

$$
D o^{2}+D i^{2}+2 l \cos \alpha D i-2 l \sin \alpha D o=0
$$

Equation (2) shows the output displacement equation:

$$
D o=l \sin \alpha-\sqrt{l^{2} \sin ^{2} \alpha-D i(D i+2 l \cos \alpha)}
$$

Displacement magnification ratio of amplifying mechanism based on triangular amplification principle is the output displacement to the input displacement. The magnification can be obtained by the formula (3):

$$
A=\frac{D o}{D i}=\frac{l \sin \alpha-\sqrt{l^{2} \sin ^{2} \alpha-D i(D i+2 l \cos \alpha)}}{D i}
$$

From the formula (4), displacement magnification of amplifying mechanism is related to the connecting rod length 1 , connecting rod angle $\alpha$ and input displacement Di. It can be seen from the calculation and analysis that the magnification A decreases with the increase of the angle $\alpha$; and the magnification A decreases with the increase of the input displacement Di. 


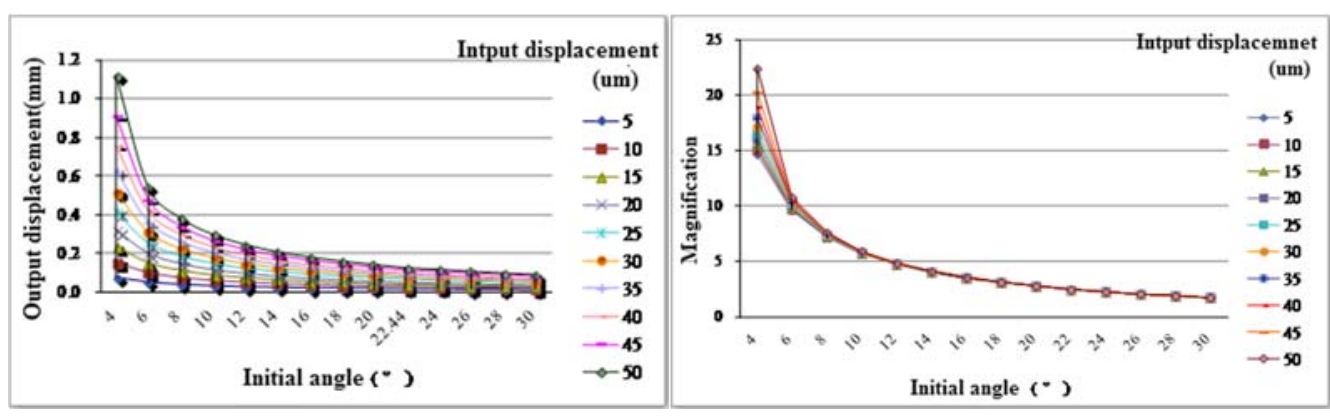

Fig.3 Relation between the output displacement Fig.4 Relation between magnification and and the initial angle of the connecting rod initial angle of connecting rod

\section{Finite Element Static Analysis of Micro-Nano Platform Based on Piezoelectric Stack}

Using actuators and hinges based on triangular amplification principle can promote the platform in the XY plane to achieve nano-level positioning. The structure shown in Figure 1. This paper uses ANSYS finite element analysis software for analysis of Micro-nano platform.

The material parameters of the amplifying mechanism in this Micro-nano platform are: 45\#steel, elastic modulus EX $=2.06 * 1011$, density $\mathrm{DENS}=7800 \mathrm{~kg} / \mathrm{m} 3$, Poisson ratio $\mathrm{PRXY}=0.27$, thickness $=0.01 \mathrm{~m}$. The material parameters of the hinge and platform are Aluminum alloy, elastic modulus $\mathrm{EX}=2.7 * 1010$, density $\mathrm{DENS}=2700 \mathrm{~kg} / \mathrm{m} 3$, Poisson ratio $\mathrm{PRXY}=0.3$, thickness $=0.01 \mathrm{~m}$. The unit type use PLANE42 (plane stress input with thickness). By mapping mesh division, the number of units is 2741 , and the number of nodes is 3328 . When the displacement of the lateral side of the fixed hinge is set to the same, and after a displacement amount of 40um is applied to the long diagonal side of the actuator composed of the piezoelectric stack 1 and amplification mechanism 2, the stress and deformation of the whole platform are obtained as shown in Figure 5 (a). The displacement of it in $\mathrm{Y}$ direction is between $92 \mathrm{um} \sim 116 \mathrm{um}$, and is very close to the analysis value $107.54 \mathrm{um}$. When two actuators are simultaneously applied with a displacement amount of $20 \mathrm{\mu} \mathrm{m}$ on the long side, the stress and deformation of the platform are obtained as shown in Figure 5 (b). Since the displacement is input at the same time in both directions, the ratio of the total displacement is no longer the same as before.

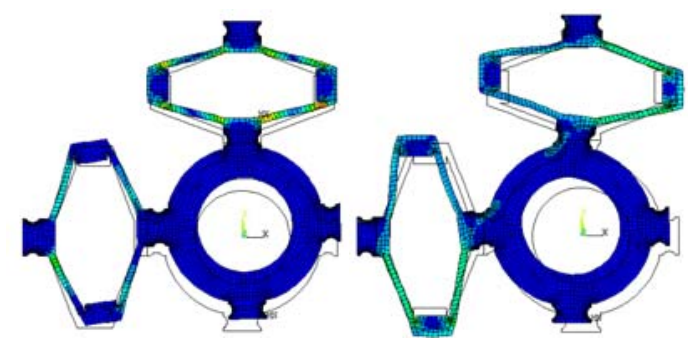

$\begin{array}{lll}\text { (a) an actuator } & \text { (b) two actuators }\end{array}$

Fig.5 Finite element analysis of the stress and deformation of micro-nano platform

From the graph, the deformation is appeared after two piezoelectric stacks are input to a certain voltage. The displacement of deformation amplified by the amplification mechanism drives the platform to output the corresponding displacement on the XY plane, and the nanometer level work localization of the platform on the XY plane is realized.

\section{The Characteristics of the Large Torque Micro-Nano Platform based on the Piezoelectric Stack}

Mode of vibration is inherent characteristics of elastic structures. Characteristics of main modes of each order are made clear by modal analysis, when the structure is in an easily affected frequency range. In the design and analysis of micro displacement platform, it is necessary to avoid its vibration mode. Therefore, the modal analysis of the micro displacement platform is carried out by using ANSYS within the range of frequency $500 \mathrm{~Hz} 30000 \mathrm{~Hz}$. The results are shown in table 1 . 
Table1. 8 order natural frequency of micro-nano platform $(\mathrm{Hz})$

\begin{tabular}{c|c|c|c}
\hline $\begin{array}{c}\text { Order of vibration } \\
\text { modes }\end{array}$ & $\begin{array}{c}\text { Resonance } \\
\text { frequency(Hz) }\end{array}$ & $\begin{array}{c}\text { Order of vibration } \\
\text { modes }\end{array}$ & $\begin{array}{c}\text { Resonance } \\
\text { frequency(Hz) }\end{array}$ \\
\hline 1 & 844.91 & 5 & 5114.3 \\
\hline 2 & 882.17 & 6 & 15825 \\
\hline 3 & 1297.3 & 7 & 19177 \\
\hline 4 & 4972.9 & 8 & 26334 \\
\hline
\end{tabular}

Transient dynamic analysis of micro displacement platform is carried out in this paper.The analysis model diagram is shown in Fig 1
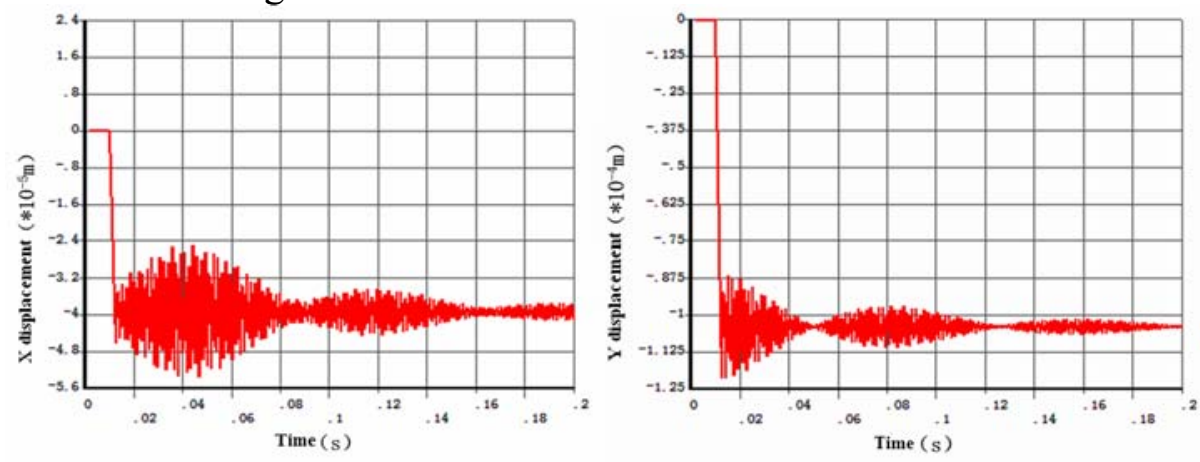

Fig. 6 the Step response of micro nano platform

Loading mode: At the $\mathrm{t}=0.01 \mathrm{~s}$ moment, load with $250 \mathrm{~N}$ pressure to edge 1 and edge 2 of the platform (as shown in Fig 1), set the camping coefficient to $5 \%$, set the step size to $0.5 \mathrm{~ms}$, the $\mathrm{X}$ direction and the $\mathrm{Y}$ direction step response characteristics of the edge 3 in the micro-nano platform are observed. The calculated results are shown in Fig 6.

\section{Conclusion}

In this paper, we design and manufacture a micro-nano platform with a high torque by using the principle of piezoelectric stack and triangular amplification. Through theoretical analysis, the displacement amplification factor of the driving part is obtained. Using finite element method analyze the displacement and stress changes of the platform. By theoretical analysis, the ineluctable hysteresis and creep characteristics of piezoelectric materials also bring some influence to the application of this platform. So characteristic testing of the micro-nano platform is needed, and the effective control methods can be used to improve the practicability of the micro-nano platform.

\section{References}

[1]. Li Cheng, Cheng-jin Zhang, Guilin Zhang. High-speed and Large-range scanning control of a piezoelectric stack actuated platform[C]. Proceedings of the 8th World Congress on Intelligent Control and Automation, Jinan, China, July 6-9 2010: 5523-5527.

[2]. Yangmin Li, Senior Member, Qingsong Xu. Development and Assessment of a Novel Decoupled XY Parallel Micropositioning Platform [J]. IEEE/ ASME Transactions on Mechatronics, 2010, 15(1): 125-135.

[3]. SUN Bao-yu. Experimental Research on Dynamic Charateristic of Flexible Micrro-displacement Mechanism Based on Piezoelectric Actuator [J]. Micro Fabrication Technology, 2008(2): 33-36.

[4]. DU Xi-bo, CHEN Xi-ping, ZHANG Bin, et al. Design and Analysis of Micro-displacement Mechanism for Motion Position with Piezo-actuator Based on Triangle Prinple[J]. Aviation Precision Manufacturing Technology, 2009(6): 10-12.

[5]. LIU Deng-yun, YANG Zhi-gang, CHENG Guang-ming, ZENG Ping. Study on a Microdisplacement Magnifying Mechanism Used on Piezo-stack Pump [J]. Machinery \& Electronics, 2007, 3:75-77. 
[6]. Li Wanquan, Gao Changyin. Design and Modeling of Micro-displacement Amplifying Mechanism Using Revolute Joints and Flexible Hinges[C]. 2010 International Conference on Intelligent Computation Technology and Automation, 2010:1147 1150.

[7]. Sebastian Polit, Jingyan Dong. Development of a High-Bandwidth XY Nanopositioning Stage for High-Rate Micro-/Nanomanufacturing [J]. IEEE/ ASME Transactions on Mechatronics, 2011, 16(4): 724-733.

[8]. Qingsong Xu, Yangmin Li, Ning Xi. Design, Fabrication, and Visual Servo Control of an XY Parallel Micromanipulator with Piezo-Actuation [J]. IEEE Transactions on Yu Songsong. Design, Analysis and Testing of a Multi-scale Micro/nano-positioning System: [D]. Chongqing University, 2011, 4. 